\title{
Infections trypanosomiennes chez des bovins dans la zone Sud-soudanienne du Burkina Faso
}

\author{
Z. Bengaly ${ }^{1}$ R. Ganaba ${ }^{1}$ I. Sidibe ${ }^{1}$ G. Duvallet ${ }^{2}$
}

\section{Mots-clés}

Bovin - Trypanosoma vivax - Trypanosoma congolense - Morbidité - Burkina Faso.

\begin{abstract}
Résumé
Afin d'évaluer la prévalence apparente des infections trypanosomiennes et quelques facteurs de variation chez les bovins dans la zone Sud-soudanienne du Burkina Faso, 1796 animaux répartis dans cinq provinces ont été prélevés entre 1994 et 1995, pour la recherche de trypanosomes par l'examen microscopique du buffy coat et du frottis sanguin coloré au Giemsa. Dans quatre des provinces prospectées en saison des pluies (juillet-août), la prévalence apparente a varié entre 7,6 p. 100 (intervalle de confiance : 4,9-11,3 p. 100) et 12,2 p. 100 (IC : 8,9-16,3 p. 100). Celle-ci a été de 4,9 p. 100 (IC: 2,78,4 p. 100) pour l'unique province où l'enquête a été effectuée en saison sèche (mars). Des facteurs de variation étudiés (province, zone d'encadrement d'élevage, phénotype animal, classe d'âge), seules la zone d'élevage et la classe d'âge semblent avoir eu un effet significatif sur la prévalence des infections chez les animaux. Une diminution en particulier de la prévalence à Trypanosoma vivax avec l'âge, alors que c'était l'inverse pour T. congolense, a été observée. L'espèce T. vivax était prédominante (64 p. 100 des infections totales), suivie par T. congolense (46,6 p. 100) et T. brucei (2,5 p. 100). II y a eu 13 p. 100 d'infections mixtes et les infections à T. vivax étaient positivement associées à celles à $\mathrm{T}$. congolense.
\end{abstract}

\section{INTRODUCTION}

La zone Sud-soudanienne du Burkina Faso est, depuis les sécheresses consécutives des années 1973-1974 et 1983-1984, une zone d'accueil des bovins du Nord et du Centre, essentiellement de race zébu, à la recherche de pâturages et d'eau (7). Or, la trypanosomose animale sévit dans ces zones humides d'Afrique de façon générale et constitue l'un des obstacles majeurs à la survie des zébus et à l'expression du potentiel de productivité des taurins et des métis qui y vivent (5). Les bases de la lutte intégrée contre cette maladie (chimiothérapie, chimioprophylaxie, contrôle des vecteurs, etc.) doivent se fonder sur la connaissance approfondie de son épidémiologie à savoir l'étude de la prévalence, sa saisonnalité et les interrelations hôte-vecteur. Les études menées au Burkina Faso à l'échelle du terroir villageois ont indiqué des prévalences de l'ordre de 4 à 25 p. 100 autour de la zone d'aménagement pastoral de Sidéradougou (3), de 80 p. 100 à Satiri (2) et enfin de 18 à 78 p. 100 à Samorogouan (1). Ces données corres-

1. Cirdes, 01 BP 454, Bobo-Dioulasso 01, Burkina Faso

Tél. : +2269722 87/972053; Fax : +226972320

2. Cirad-emvt, Campus international de Baillarguet, BP 5035, 34032 Montpellier Cedex 1, France pondent à des situations épidémiologiques particulières (forte pression glossinienne liée à l'existence de forêts classées et de galeries forestières, présence de souches de trypanosomes chimiorésistants, présence importante de zébus) et ne peuvent refléter celles de toute la zone Sud-soudanienne. La présente étude a été réalisée pour recueillir des données complémentaires sur la prévalence de la trypanosomose et quelques facteurs de sa variation dans l'ensemble de cette zone à travers des sondages ponctuels effectués ces dernières années.

\section{MATERIEL ET METHODES}

La zone Sud-soudanienne, située entre 5 et $12,5^{\circ}$ de latitude Nord et 0,5 et $5,5^{\circ}$ de longitude Ouest, représente 32 p. 100 du territoire national. Elle est composée de huit provinces dont chacune est divisée en six ou huit zones d'encadrement de l'élevage. Les glossines rencontrées sont Glossina tachinoides, G. palpalis gambiensis (le long des galeries forestières), G. morsitans submorsitans (dans la savane) et $G$. medicorum (localisée dans les grosses formations végétales à la frontière avec la Côte d'Ivoire) (4).

Le choix des animaux a été effectué selon un mode d'échantillonnage à plusieurs niveaux (zones, villages, troupeaux, individus). Trois à quatre zones d'encadrement de l'élevage ont été choisies 
dans chacune des cinq provinces étudiées (figure 1). Dans chaque zone au moins deux villages ont été retenus. Environ un tiers des troupeaux a été sélectionné dans chaque village. A chacune de ces étapes, les unités d'échantillonnage ont été choisies selon le mode aléatoire simple. Enfin, dans chaque troupeau, les animaux ont été soumis à l'échantillonnage systématique. Le nombre d'animaux a été défini selon le mode d'allocation proportionnelle. Au total, 1796 bovins appartenant à 169 troupeaux ont été examinés. Leur répartition, en fonction des provinces, est présentée dans le tableau I.

Les prélèvements sanguins ont été effectués à l'aide de tubes contenant un anticoagulant (héparine), au niveau de la veine jugulaire, pour la recherche de trypanosomes. Le sang collecté a été soumis sur place à une centrifugation différentielle dans un microtube capillaire. Puis, le microtube a été sectionné et le buffy coat a été examiné en microscopie à fond noir (grossissement x 400) après un étalement entre lame et lamelle (10). En outre, un étalement de sang total a été réalisé systématiquement pour être examiné au laboratoire après fixation au méthanol et coloration au Giemsa (12) afin de compléter les examens parasitologiques réalisés sur le terrain.

Tous les prélèvements ont été effectués en saison des pluies (juillet à septembre 1994), à l'exception de la province de la Comoé où ils ont été effectués en saison sèche (mars 1995), pour des raisons d'inaccessibilité.

Les analyses statistiques ont consisté à calculer les prévalences des infections et à en déterminer les intervalles de confiance (IC) au seuil de 95 p. 100. Les comparaisons statistiques des prévalences ont été faites selon le test de correction de continuité des intervalles (13). Un animal est considéré infecté si l'examen de son sang a révélé la présence de trypanosomes pathogènes par l'examen du buffy coat ou par celui du frottis coloré. L'association de la présence des différentes espèces de trypanosomes chez les animaux a été estimée par la méthode loglinéaire.

\section{RESU LTATS ET DISCU SSIO N}

La prévalence apparente des infections trypanosomiennes chez les bovins était peu élevée à l'échelle de l'ensemble de la population étudiée et sujette à une faible variation d'une province à l'autre. Par contre, une variation très importante a été souvent obtenue entre des zones d'encadrement de l'élevage appartenant à une même province (tableau II).

Cette variation pouvait être liée à l'utilisation peu ou très répandue d'insecticides contre les glossines et, ou des trypanocides en fonction de la province et surtout de la zone d'encadrement de l'élevage. Dans la zone de Samorogouan, la faible prévalence observée pouvait résulter des traitements épicutanés des animaux par pouron à l'aide d'un insecticide (deltaméthrine : 1 p. 100) institués par les communautés rurales depuis la mise en place d'une campagne de lutte antivectorielle de 1989 à 1992 au Ceziet * (1). Dans les zones de Banfora et de Founzan, la plupart des troupeaux avaient dû être traités avec un trypanopréventif peu avant l'examen des prélèvements sanguins car les éleveurs des sites sélectionnés respectaient rigoureusement les calendriers thérapeutiques préconisés par les techniciens d'élevage pour lutter contre les principales contraintes sanitaires dont la trypanosomose. La diversité probable des différentes espèces de glossines présentes sur les sites et des systèmes d'élevage (transhumant ou sédentaire) pouvaient également avoir influé sur les prévalences trypanosomiennes obtenues, mais leur impact n'a pu être évalué.

Les prévalences obtenues dans cette étude étaient faibles par rapport à celles rapportées à Satiri (2) et à Samorogouan (1). Ceci pouvait être lié aux conditions épidémiologiques particulières caractérisées par une forte pression glossinienne notamment due à Glossina morsitans submorsitans et à la prédominance d'animaux de race zébu présumés trypanosensibles au niveau de ces deux sites au moment des investigations précédentes.

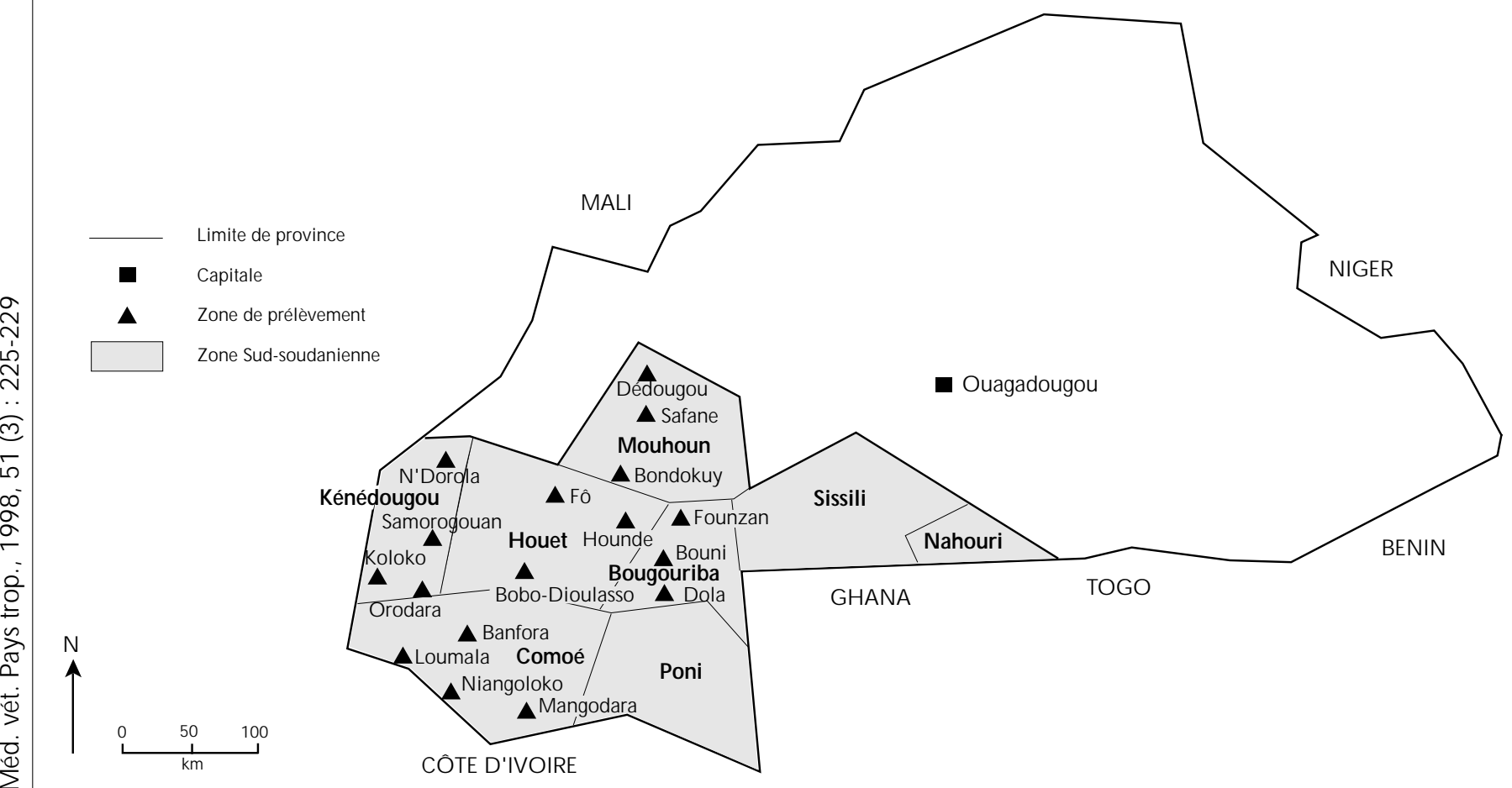

Figure 1 : zone Sud-soudanienne du Burkina Faso. 
Tableau I

Répartition des animaux échantillonnés dans cinq provinces de la zone Sud-soudanienne du Burkina Faso

\begin{tabular}{|c|c|c|c|c|c|c|}
\hline \multirow[b]{2}{*}{ Zone } & \multicolumn{5}{|c|}{ Province } & \multirow[b]{2}{*}{ Total } \\
\hline & Bougouriba & Comoé & Houet & Kenedougou & Mouhoun & \\
\hline Dolo & 123 & & & & & \\
\hline Guéguéré & 55 & & & & & \\
\hline Founzan & 151 & & & & & \\
\hline Banfora & & 55 & & & & \\
\hline Loumana & & 65 & & & & \\
\hline Niangoloko & & 39 & & & & \\
\hline Mangodara & & 105 & & & & \\
\hline Bobo-Dioulasso & & & 32 & & & \\
\hline Houndé & & & 107 & & & \\
\hline Fô & & & 131 & & & \\
\hline Padema & & & 122 & & & \\
\hline Orodara & & & & 181 & & \\
\hline Koloko & & & & 83 & & \\
\hline Samorogouan & & & & 97 & & \\
\hline $\mathrm{N}$ dorola & & & & 148 & & \\
\hline Dédougou & & & & & 85 & \\
\hline Bondoukuy & & & & & 83 & \\
\hline Safane & & & & & 134 & \\
\hline Total (nb. d'animaux) & 329 & 264 & 392 & 509 & 302 & 1796 \\
\hline M étis (Baoulé x Zébu) & 92 & 135 & 112 & 168 & 230 & 737 \\
\hline Baoulé & 14 & 53 & 58 & 30 & 02 & 157 \\
\hline Zébu & 223 & 76 & 222 & 311 & 70 & 902 \\
\hline$<1$ an & 45 & 18 & 08 & 38 & 07 & 116 \\
\hline $1-2$ ans & 101 & 58 & 57 & 105 & 49 & 370 \\
\hline$>2$ ans & 182 & 182 & 323 & 325 & 246 & 1258 \\
\hline ND & 01 & 06 & 04 & 41 & & 52 \\
\hline Mâle & 111 & 57 & 169 & 145 & 48 & 530 \\
\hline Femelle & 217 & 200 & 217 & 341 & 253 & 1228 \\
\hline ND & 01 & 07 & 06 & 23 & 01 & 38 \\
\hline
\end{tabular}

ND : non déterminé

L'étude des facteurs intrinsèques de variation a montré que le phénotype animal n'a pas semblé influer sur la prévalence apparente des infections trypanosomiennes. Les animaux de phénotype zébu ont semblé plus infectés que les Baoulés et les Métis mais les différences observées n'étaient pas significatives (tableau III). Cela pouvait suggérer que le risque trypanosomien était le même pour ces trois phénotypes mais que ceux qui avaient présenté de faibles prévalences apparentes étaient plus aptes à contrôler la parasitémie.

En revanche, la prévalence trypanosomienne a semblé diminuer avec l'âge des animaux. L'immunité acquise progressivement avec l'âge pouvait constituer un facteur déterminant. En particulier, une diminution de la prévalence à Trypanosoma vivax a été observée avec l'âge, alors que c'est l'inverse qui a été observé avec T. congolense (tableau III).

La prédominance des infections à $T$. vivax chez les veaux a déjà été observée au Burkina Faso dans la zone d'aménagement pastoral de Sidéradougou (6). Des observations similaires ont également été rapportées au Nord de la Côte d'Ivoire (11) et au Zaïre (9).
Ce phénomène pouvait être dû à l'immunité acquise progressivement avec l'âge en particulier contre l'espèce $T$. vivax en raison de sa variabilité antigénique plus limitée comparée à celle de $T$. congolense (12). L'influence du mode de conduite des veaux, caractérisé par la mise au piquet ou le gardiennage à proximité des habitations qui réduit ainsi leur contact avec des gîtes potentiels de glossines vectrices de $T$. congolense (savanes boisées et galeries forestières), était également probable.

A l'échelle de la population étudiée, $T$. vivax était prédominant (64 p. $100 \mathrm{du}$ total), suivi par T. congolense (46,6 p. 100) et T. brucei (2,5 p. 100). L'analyse des infections mixtes (13 p. 100 du total) a montré que la détection d'une infection à $T$. vivax était positivement associée à celle de $T$. congolense. La prédominance de $T$. vivax sur les autres espèces de trypanosomes et son association fréquente avec $T$. congolense ont été observées chez les bovins du pays Lobi à l'extrême Sud-Ouest du Burkina Faso (Bassinga et coll., en préparation). La forte prévalence de $T$. vivax comparée à celle de $T$. congolense suggère l'importance du contact entre les animaux et, soit les glossines du groupe palpalis connues comme des vecteurs efficaces de $T$. vivax (8), soit d'autres insectes piqueurs connus comme vecteurs mécaniques. 


\section{Tableau II}

Prévalence apparente des infections trypanosomiennes chez les bovins en fonction de la province et de la zone d'encadrement d'élevage

\begin{tabular}{|c|c|c|c|}
\hline Province & $\begin{array}{c}\text { Prévalence par province } \\
\% \text { (IC) }\end{array}$ & Zone d'encadrement d'élevage & $\begin{array}{l}\text { Prévalence par zone } \\
\% \text { (IC) }\end{array}$ \\
\hline Bougouriba & $12,2(8,9-16,3)$ & $\begin{array}{l}\text { Dolo } \\
\text { Guéguéré } \\
\text { Founzan }\end{array}$ & $\begin{array}{c}20,3(13,8-8,7) \\
21,8(12,2-35,3) \\
2,0(0,5-6,1)\end{array}$ \\
\hline Comoé & $4,9(2,7-8,4)$ & $\begin{array}{c}\text { Banfora } \\
\text { Loumana } \\
\text { Niangoloko } \\
\text { Mangodara }\end{array}$ & $\begin{array}{l}0,0(0-8,1) \\
9,2(3,8-19,6) \\
7,7(2-21,9) \\
3,8(1,2-10,0)\end{array}$ \\
\hline Houet & $9,2(6,5-12,5)$ & $\begin{array}{l}\text { Bobo-D sso } \\
\text { Houndé } \\
\text { Fô } \\
\text { Padema }\end{array}$ & $\begin{array}{r}9,4(2,4-26,1) \\
4,7(1,7-11,0) \\
13,7(8,5-21,1) \\
8,2(4,2-14,9)\end{array}$ \\
\hline Kenedougou & $9,6(7,2-12,6)$ & $\begin{array}{c}\text { Orodara } \\
\text { Koloko } \\
\text { Samorogouan } \\
\text { N dorola }\end{array}$ & $\begin{array}{c}12,8(8,4-18,7) \\
19,3(11,7-29,7) \\
1,0(0-6,4) \\
6,0(2,9-11,4)\end{array}$ \\
\hline Mouhoun & $7,6(4,9-11,3)$ & $\begin{array}{l}\text { Dédougou } \\
\text { Bondoukuy } \\
\text { Safane }\end{array}$ & $\begin{array}{c}16,5(9,6-26,4) \\
3,6(0,9-10,9) \\
4,5(1,8-9,9)\end{array}$ \\
\hline Total & $9(7,7-10,4)$ & - & - \\
\hline
\end{tabular}

IC : intervalle de confiance

Tableau III

Prévalence apparente des infections trypanosomiennes chez les bovins en fonction du phénotype et de la classe d'âge

\begin{tabular}{|c|c|c|c|c|c|c|c|}
\hline & \multirow{2}{*}{$\begin{array}{c}\text { Espèce de } \\
\text { trypanosomes }\end{array}$} & \multicolumn{3}{|c|}{ Phénotype animal } & \multicolumn{3}{|c|}{ Classe d'âge } \\
\hline & & $\begin{array}{l}\text { Métis } \\
\text { (IC) }\end{array}$ & $\begin{array}{l}\text { Zébu } \\
\text { (IC) }\end{array}$ & $\begin{array}{l}\text { Baoulé } \\
\text { (IC) }\end{array}$ & $\begin{array}{c}<1 \text { an } \\
\text { (IC) }\end{array}$ & $\begin{array}{l}\text { 1-2 ans } \\
\text { (IC) }\end{array}$ & $\begin{array}{c}>2 \text { ans } \\
\text { (IC) }\end{array}$ \\
\hline \multirow[t]{3}{*}{$\begin{array}{l}\text { Prévalence } \\
\text { spécifique \% }\end{array}$} & T. brucei & $\begin{array}{c}0 \\
(0-0,6)\end{array}$ & $\begin{array}{c}0,4 \\
(0,1-12,1)\end{array}$ & $\begin{array}{c}0,6 \\
(0-4,0)\end{array}$ & $\begin{array}{c}0 \\
(0-0,07)\end{array}$ & $\begin{array}{c}0 \\
(0,02-1,2)\end{array}$ & $\begin{array}{c}0,07 \\
(0,0-0,5)\end{array}$ \\
\hline & T. congolense & $\begin{array}{c}4,9 \\
(3,4-6,7)\end{array}$ & $\begin{array}{c}3,9 \\
(2,7-5,4)\end{array}$ & $\begin{array}{c}1,9 \\
(0,4-5,9)\end{array}$ & $\begin{array}{c}3,4 \\
(1,1-9,1)\end{array}$ & $\begin{array}{c}0,8 \\
(0,02-2,5)\end{array}$ & $\begin{array}{c}4,8 \\
(3,6-6,1)\end{array}$ \\
\hline & T. vivax & $\begin{array}{c}2,9 \\
(1,9-4,5)\end{array}$ & $\begin{array}{c}5,4 \\
(4,0-7,1)\end{array}$ & $\begin{array}{c}7 \\
(3,7-12,4)\end{array}$ & $\begin{array}{c}15,5 \\
(9,7-23,6)\end{array}$ & $\begin{array}{c}6,2 \\
(4-9,3)\end{array}$ & $\begin{array}{c}4,5 \\
(3,4-5,8)\end{array}$ \\
\hline $\begin{array}{l}\text { Prévalence } \\
\text { totale } \%\end{array}$ & - & $\begin{array}{c}7,7 \\
(5,9-9,9)\end{array}$ & $\begin{array}{c}10 \\
(8,1-10,1)\end{array}$ & $\begin{array}{c}8,9 \\
(5,1-14,7)\end{array}$ & $\begin{array}{c}16,4 \\
(10,3-14,6)\end{array}$ & $\begin{array}{c}7,8 \\
(5,3-11,1)\end{array}$ & $\begin{array}{c}8,3 \\
(6,9-10)\end{array}$ \\
\hline
\end{tabular}

IC : intervalle de confiance

\section{CONCLUSION}

Cette étude a montré une faible variation de la prévalence apparente de la trypanosomose chez les bovins de la zone Sud-soudanienne entre les provinces et entre les phénotypes qui y résidaient. La variation n'était importante qu'entre certaines zones d'encadrement d'élevage appartenant à une même province et entre certaines classes d'âge. A l'échelle de l'ensemble de la population, la présence d'une infection à $T$. vivax était positivement associée à celle à $T$. congolense.
Les investigations doivent être approfondies par une étude de l'incidence de la trypanosomose, son impact sur les performances de productivité des bovins et les implications socio-économiques et culturelles, particulièrement à l'échelle des terroirs bien ciblés. Les données recueillies serviront de base à l'élaboration de stratégies de contrôle de la maladie, indispensables à toute promotion de l'élevage dans cette zone.

\section{Remerciements}

Nous tenons à remercier la section Santé et production animale de l'Aiea et le Cirad-emvt pour le financement de cette étude. Nous 
exprimons notre gratitude aux docteurs R. Dwinger et D. Cuisance pour leur correction du manuscrit, leurs conseils et appuis. Ce travail est le fruit de la collaboration avec les Services provinciaux des ressources animales (Spra) du Burkina Faso auxquels nous adressons nos sincères remerciements.

\section{BIBLIO GRAPHIE}

1. BAUER B., AMSLER-DELAFOSSE S., CLAUSEN P.H., KABORE I., PETRICH-BAUER J., 1995. Successful application of deltamethrin pouron to cattle in a campaign against tsetse flies Glossina spp. in the pastoral zone of Samorogouan. Trop. Med. Parasitol., 46: 183-189.

2. BAUER B., KABO RE I., LIEBISCH A., MEYER F., PETRICH-BAUER J., 1992. Simultaneous control of ticks and tsetse flies in Satiri, Burkina Faso, by the use of flumethrin pour-on for cattle. Trop. Med. Parasitol., 43: $41-46$

3. BAUER B., PETRICH-BAUER J., KABORE I., KOUROUMA B., MATTAUSCH M., SOME J., TAMBOURA I., 1988. Epidemiological survey in the pastoral zone of Sideradougou, Burkina Faso. In: Proc. int Symp. on Modern insect control (Nuclear techniques and biotechnology), Vienna, Austria, 16-20 November 1987. Vienna, Austria, AIEA, p. 139-149.

4. CHALLIER A., LAVEISSIERE C., 1977. La répartition des glossines en Haute-Volta. Une carte couleur au $1 / 200000^{\mathrm{e}}$ et notice explicative. Paris, France, O rstom

5. FAO, 1983. La trypanosomiase animale africaine. Rome, Italie, Fao, 78 p. (Production et santé animale, vol. 37)

6. GAUTHIER C., 1996. Contribution à la connaissance de la situation épidémiologique de la trypanosomose bovine dans la zone de Sidéradougou. Mém. Dess Productions animales en région chaude, Cirad-emvt, Montpellier, France, $53 \mathrm{p}$.

\section{Summary}

Bengaly Z., Ganaba R., Sidibe I., Duvallet G. Trypanosome infections in cattle in the South-Sudanese area of Burkina Faso

In five provinces of the South-Sudanese area of Burkina Faso 1796 cattle were sampled between 1994 and 1995 for the presence of trypanosome infections using the buffy coat technique and Giemsa stained blood smears. The apparent prevalence of trypanosome infections and some variation factors were assessed. The investigations took place during the rainy season (July-August) in four provinces, where prevalence rates varied between 7.6 and $12.2 \%(4.9-11.3 \%$ and $8.9-16.3 \%$ confidence intervals, respectively). In the only province investigated during the dry season ( $\mathrm{March}$ ) the prevalence was $4.9 \%(2.7-8.4 \% \mathrm{Cl})$. Of the studied variation factors (province, livestock monitored area, animal phenotype and age group), only the livestock area and age group showed a significant effect on the infection prevalence in the animals. Particularly, a decrease of T. vivax prevalence was associated with age, while the opposite was observed with $\mathrm{T}$. congolense. T. vivax was the most common species $164 \%$ of total infections), followed by $\mathrm{T}$. congolense $(46.6 \%)$ and $\mathrm{T}$. brucei $(2.5 \%)$. There were $13 \%$ mixed infections and $\mathrm{T}$. vivax infections were positively associated with $\mathrm{T}$. congolense's.

Key words: Cattle - Trypanosoma vivax - Trypanosoma congolense - Morbidity - Burkina Faso.
7. M.A.R.A., 1989. Etude prospective du sous-secteur élevage au Burkina Faso. Rapport de synthèse, tome I. Paris, France, Ministère de l'Agriculture et des ressources animales, $364 \mathrm{p}$.

8. MOLOO S.K., KUTUZA S.B., 1988. Comparative study on the infection rates of different laboratory strains of Glossina species by Trypanosoma congolense. Med. vet. Entomol., 2: 253-257.

9. MULUNGO M., D'IETEREN G., FERON A., ITTY P., MAEHL M., NAGDA S., PALING P., RARIEYA M., THORPE W., TRAIL J.C.M., 1987. La trypanosomiase chez les bovins N'dama au Zaïre et ses effets sur la santé et la production. In : XIVe réunion du conseil scientifique pour la recherche et la lutte contre la trypanosomiase, Lomé, Togo, 30 mars 3 avril 1987, p. 530-533

10. MURRAY M., MURRAY P.K., MCINTYRRE W.M., 1977. An improved parasitological technique for the diagnosis of African trypanosomiasis. Trans. R. Soc. trop. Med. Hyg., 71: 325-326.

11. SCHUTTERLE A., COULIBALY L., DIARRASSO UBA I., D'IETEREN G., ITTY P., KONIN N., MAEHL M., MAHAMAT B., NAGDA S., PALIN G P., RARIEYA M., THORPE W., TRAIL J.C.M., 1987. L'influence des infections trypanosomiennes sur les paramètres sanitaires et zootechniques au nord de la Côte d'Ivoire. In : XIVe réunion du conseil scientifique pour la recherche et la lutte contre les trypanosomiases, Lomé, Togo, 30 mars - 3 avril 1987, p. 521-525.

12. TRO NCY P.M., ITARD J., M O REL P.C., 1981. Précis de parasitologie vétérinaire tropicale. Paris, France, Ministère de la coopération et du développement, 717 p. (Manuels et précis d'élevage)

13. VOLLSET S.E., 1993. Confidence intervals for the binomial proportion. Stat. Med., 12: 809-824.

Reçu le 13.1.98, accepté le 18.11 .98

\section{Resumen}

Bengaly Z., Ganaba R., Sidibe I., Duvallet G. Infecciones tripanosómicas en bovinos en la zona sud sudanese de Burkina Faso

Con el fin de evaluar la prevalencia aparente de infecciones tripanosómicas y de algunos factores de variación en los bovinos en la zona Sud sudanese de Burkina Faso, se tomaron muestras de 1796 animales, distribuidos en cinco provincias, entre 1994 y 1995, para identificar tripanosomas mediante el examen microscópico de buffy coat y por frotis sanguíneo con colorante de Giemsa. En cuatro de las provincias estudiadas durante la estación lluviosa (julio-agosto), la prevalencia aparente varió entre 7,6\% (intervalo de confianza: 4,9-11,3\%) y $12,2 \%$ (IC: $8,9-16,3 \%$ ). Esta fue de $4,9 \%$ (IC: $2,7-8,4 \%$ ) en la única provincia en que la encuesta se efectuó durante la estación seca (marzo). Los factores de variación estudiados (provincia, zona de producción, fenotipo animal, rango de edad), únicamente la zona de producción y el rango de edad parecen tener un efecto significativo sobre la prevalencia de las infecciones en los animales. En particular, se observó una disminución de la prevalencia de las infecciones por Trypanosoma vivax con la edad, inversamente a las de T. congolense. La especie T. vivax fue predominante $(64 \%$ del total de las infecciones), seguida por $T$. congolense $(46,6 \%)$ y T. brunei $(2,5 \%)$. Hubo $13 \%$ de infecciones mixtas y las infecciones por T. vivax estuvieron positivamente asociadas a las de $T$. congolense.

Palabras clave: Ganado bovino - Trypanosoma vivax - Trypanosoma congolense - Morbosidad - Burkina Faso. 